\title{
Evaluating Benefits of Rolling Horizon Model Predictive Control for Intraday Scheduling of a Natural Gas Pipeline Market
}

\author{
Aleksandr Rudkevich \\ Newton Energy Group \\ arudkevich@negll.com \\ Pablo A. Ruiz \\ Boston University \\ paruiz@bu.edu \\ Richard Tabors \\ Tabors Caramanis Rudkevich \\ rtabors@tcr-us.com
}

\author{
Anatoly Zlotnik \\ Los Alamos National Laboratory \\ azlotnik@lanl.gov \\ Aleksandr Beylin \\ Newton Energy Group \\ abeylin@negll.com \\ Russ Philbrick \\ Polaris System Optimization \\ russ.philbrick@psopt.com
}

\author{
Xindi Li \\ Tabors Caramanis Rudkevich \\ xindi@tcr-us.com \\ Evgeniy Goldis \\ Newton Energy Group \\ jgold@negll.com
}

\begin{abstract}
This paper analyzes a mechanism for clearing a physical market for intra-day schedules of receipts and deliveries of a natural gas pipeline. The Gas Balancing Market (GBM) is implemented to trade deviations from previously confirmed ratable nominations by solving a rolling horizon model predictive control (MPC) optimization formulation. The GBM mechanism operates by accepting quantity/price offers and bids from sellers and buyers of gas and producing an economically optimal schedule while guaranteeing its physical feasibility. The GBM's solution engine is based on a strict mathematical representation of engineering factors of transient pipeline hydraulics and compressor station operations. The GBM's settlement of cleared transactions is based on Locational Trade Values (LTVS) of natural gas that are fully consistent with the physics of energy flow. In this paper we provide numerical results of simulating a hypothetical GBM market operation using historical SCADA data for an actual pipeline system operation during the Polar Vortex period of February - March 2014. Based on these simulations, we quantify the potential deliverability and economic benefits of the GBM utilizing transient optimization of pipeline operations.
\end{abstract}

\section{Introduction}

The growing reliance of the bulk electric power system on gas-fired generation has increased the need to improve the coordination between the wholesale natural gas and electricity markets.

Replacement of coal fired and nuclear plants with gas-fired generating capacity significantly increases the amount of natural gas used as fuel for power generation. At the same time, the volatility of electric generation delivered from wind and solar increases the variability of pipeline deliveries to match the demand of gas-fired generators used to balance the electric grid. The resulting intra-day and even sub-hourly demand swings for natural gas to fuel for electric generation create new challenges for pipeline operators, and may pose reliability risks for both gas pipelines and electric systems.

The need to better coordinate both sectors to mitigate these risks is well recognized and is specifically reflected in Orders 787 and 809 of the Federal Energy Regulatory Commission (FERC) that regulates access to pipeline capacity [1,2]. Coordination mechanisms proposed to date are based on widening the scope of operational information exchanges between the two sectors, and on adjusting the timing of these exchanges [3]. While these measures are helpful, a truly efficient coordination should be based on timely exchange of both physical and pricing data, with price formation in both markets being fully consistent with the physics of energy flow.

Electricity prices consistent with the physical power flows on the grid are the outcome of economic optimization of power system operation in electricity markets administered by Regional Transmission Organizations (RTOs) [4, 5]. A similar optimization approach that accounts for physical and engineering 
factors of gas pipeline and compressor station operations would lead to location and time dependent economic valuation of natural gas consistent with the physics of gas flow. Such an approach has been formulated in [6] under the simplified assumption of steady state pipeline flows.

A more general formulation would consider a truly dynamic transient flow of natural gas, which is essential for representing gas flow dynamics in time frames of the same order of physical changes that occur on the power grid. The problem formulation can be described as a model predictive control (MPC) approach, which uses a forecast of system conditions and other time-dependent inputs to optimize pipeline flows over a given time-horizon, such as 24 hours.

This paper relies on [8, 14]. Ref. [8] defines a transient pipeline optimization problem that maximizes total market surplus over supply and offtake schedules. The market surplus in defined within the context of Gas Balancing Market [14]. Market Surplus is defined as the sum of the producer/supplier surplus and consumer/buyer surplus. Producer surplus is the difference between the revenue the producer obtains in the market and the minimum value the producer is willing to accept for the goods sold. Similarly, consumer surplus is the difference between the maximum price the consumer is willing to pay for a good and the price the consumer ends up paying for it. Market surplus is the sum of individual surpluses over all consumers/buyers and producers/sellers participating in the market.

The appropriate transient optimization solution dynamically allocates pipeline capacity among transactions between suppliers and consumers based on the economic value of these transactions. Compressor operations and line pack are optimized in conjunction with the selection of location-dependent offers to sell, and bids to buy natural gas. Location based (nodal) prices of natural gas are computed as dual variables corresponding to the nodal flow balance constraints in the optimal solution and reflect the time and location dependent economic value of gas in the network.

In this paper we demonstrate the capability to operate a pipeline system in practice using the Gas Balancing Market (GBM) introduced in [14] through a case study of a section of a pipeline subsystem in the United States using network data obtained from a capacity planning model as well as physical supervisory control and data acquisition (SCADA) system data and historical market information during a period of highly congested conditions. We demonstrate that using our proposed formulation it is possible to significantly increase pipeline throughput with the added benefit of more efficient prices throughout the system. Both advantages are demonstrated with respect to historical data on physical and market conditions. In addition to quantifying the economic benefits of the proposed mechanism, we demonstrate the potential to use the modeling tool in analysis and planning of wholesale electricity markets with high reliance on natural gas.

This is the first paper to present an analysis of the operation of the natural gas market design based on transient optimization of a pipeline network utilizing real system data. In addition to incorporating actual physical data we developed a set of economic inputs based on actual economic information and on assumptions made to compensate for the lack of data needed to model the behavior of market participants.

To place simulation results into an appropriate context, Section 2 restates the motivation and key principles underlying the proposed Gas Balancing Market (GBM).

Section 3 provides and details of the computational case study using real data, Section 4 provides analysis of simulation results and discusses some policy implications. Section 5 contains a summary of conclusions.

\section{Gas Balancing Market}

\subsection{Motivation}

Electric and natural gas networks follow distinct but inter-related decision processes for scheduling their operations. As discussed in [14], there exists a highly intricate succession of decision cycles on both the electric side and natural gas side. A gas-fired generating unit considering operating the next electric day (which begins at midnight) should submit an offer to the Day-Ahead power market on the prior day by 10:30 AM Eastern Time. Prior to that, the asset manager for the generating unit would procure gas supply and delivery. Because electric generators usually do not have firm supply contracts on the pipeline, delivery to a pipeline receipt point will be arranged at a bilaterally negotiated price. Shipment of gas from the receipt point to the delivery point on the pipeline could be arranged on a firm basis through the capacity release mechanism or on a non-firm basis by obtaining interruptible capacity. This process yields a preliminary supply arrangement and gas prices. These prices, although not backed up by delivery guarantees, inform electric generators on how to bid in the dayahead (DA) electricity market. This process exposes transacting parties to various kinds of risk.

Once the DA market clears and the financially binding operational schedules for electric generators are determined, generators have just enough time to 
make delivery nominations with the pipeline for the next gas day. If the nominations are confirmed in the Timely and/or Evening gas scheduling cycles, daily delivery quantities are essentially guaranteed. However, even if confirmed, the quantities needed by the generator may be different from those preliminarily arranged and the difference must be settled between the parties.

If deliveries needed by the generator are not confirmed due to pipeline capacity limitations, generators would face significant financial exposure when they are obligated to deliver power but have no gas to produce it. This financial exposure is two-fold: the generator may need to acquire replacement power in the real-time market and also may be facing nonperformance penalties if the electric replacement power purchase occurs at a time of scarcity.

Even if the daily delivery quantity is confirmed, the pipeline typically expects that gas will be taken in equal quantities each hour of the gas day (i.e., a "ratable" quantity). Generators, however, typically need non-ratable quantities which pipelines may be able to accommodate but cannot guarantee.

Furthermore, most fast-start combined cycle generators and gas turbine peaking facilities are not committed in the DA market. Instead those units are typically scheduled through the hourly reliability updates or close to the real-time market. These "lastminute" decisions do not fit into the existing decision cycles on the gas side. For these generators that are critical for maintaining a reliable electric service and providing essential ancillary services there is no transparent mechanism on the gas side under which they can purchase gas and schedule delivery as needed. Sudden ramps required by these generators may cause operational problems to the pipelines. If these generators receive no gas, this would jeopardize the operational reliability of the electrical grid, while delivering gas to these units may jeopardize the reliability of the pipeline system.

We propose to solve these problems through the formation of the hour-by-hour natural gas balancing market (GBM) that would allow market participants to trade deviations from approved ratable schedules throughout the Timely and Evening Cycles.

These deviations could be traded through the formal optimization-based auction type market mechanisms described in the next section. Such an auction could be run on an hourly basis using a rolling horizon approach, such that each hour the auction would optimize the system for multiple upcoming hours (e.g. 36 hours or even more). Such a balancing market would provide a repeated forward-looking price discovery mechanism to help both the gas and electric sectors to more efficiently coordinate their operations.

\subsection{Gas Balancing Market}

The proposed Gas Balancing Market (GBM) would have voluntary participation and would be administered by a pipeline specific market operator. It would function as a two-sided auction conducted on the gas pipeline network.

GBM transactions occur at network nodes that are primarily custodial meters where gas changes hands, compressor stations, and pipe interconnection points. Network edges are the pipes that physically connect the nodes. Auction participants are buyers and sellers of gas submitting price/quantity (P/Q) offers/bids to sell/buy gas at network nodes. Their buy/sell positions are primarily driven by the need to buy gas above the ratable schedules or the desire to sell gas in excess of ratable schedules. Thus, the same market participant may act as a buyer in one hour and as a seller in another hour. Ratable schedules would be based on deliveries confirmed at the Timely or Evening nomination cycles. In addition, the market would allow participation of buyers and sellers who have no day-ahead confirmed schedules or firm capacity rights.

Offers and bids are submitted with an hourly time step for a multi-hour optimization horizon (e.g., 36 hours).

The Auctioneer's objective function is to maximize, over the optimization horizon, the market surplus between accepted bids and offers less the costs of running the pipeline by operating gas compressors.

The auction repeats periodically (e.g. every hour or every several hours based on actual design) with the start and end time of the optimization horizon shifting along with the auction repeat time and continuously transitioning from one gas day to another.

The optimization problem is formulated subject to the dynamic transient pipeline flow equations and must satisfy key engineering constraints. The latter are limitations on the maximum allowed operating pressure at each pipe, minimum pressure requirements at each node, horsepower limitations and compression ratios of compressors.

The outcome of the auction includes:

- Hourly schedules for receipt and deliveries of natural gas over the optimization horizon for each buyer and seller and for each node of the network. These schedules are the net results of ratable schedules and buy/sell positions cleared in the market;

- Hourly shadow prices of nodal mass balance equations referred to as Locational Trade Values (LTVs) of natural gas;

- Operational compressor setting and compression ratios for each compressor station; 
- Pressure dynamics at pipes and nodes.

Market clearing for the first hour of the optimization horizon will be ex post to actual deliveries in that hour.

Market clearings for all subsequent hours of that horizon will be ex ante.

All cleared positions for all hours of the optimization horizon will be financially binding.

These market results will be financially binding, thus giving gas buyers and sellers assurance of obtaining needed gas or liquidating any excess supply at a price that is acceptable and known ahead of the delivery. Since the market is cleared multiple times for the same period, future uncertainties are resolved. This is because the positions taken in earlier instances of the GBM could be liquidated later if necessary or desirable. In sum, the proposed market structure will provide a transparent and efficient mechanism for near real-time gas trading and corresponding gas price discovery.

The proposed GBM timing is aligned with nomination cycles that are in place for natural gas pipelines in the United States. The first trading of a particular GBM cycle is aligned with the completion of the Evening Nomination Cycle (21:00 Central Time) and would have an optimization horizon of 36 hours which will cover the remaining portion of the current gas day (from 21:00 to 09:00 of the following day) and the entire gas day from 09:00 to 09:00 of the day that follows. The next trading will occur at 22:00 and will have an optimization horizon of 35 hours also ending at 09:00 of the day after the following day.

Because the optimization is conducted subject to the gas flow physics and engineering constraints, market clearing assures feasibility of delivery schedules identified. The underlying mathematical formulation of the optimization problem behind GBM is provided in [14].

\subsection{Gas System Optimizer (GSO)}

The study used our Gas System Optimizer (GSO) model schematically depicted in Figure 1. GSO implements transient optimization algorithms as described in [12].

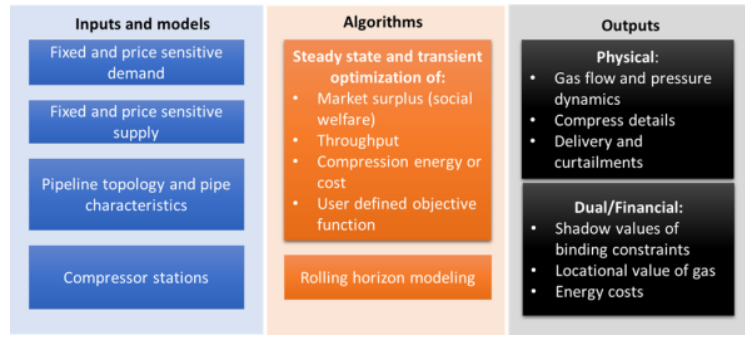

Figure 1. Gas Supply Optimizer
GSO optimizes the dynamic schedules and operation of a natural gas pipeline network. GSO finds the optimal pipeline flows and delivery schedules with the concurrent operations of compressor stations and line pack dynamics. In parallel, GSO determines the economic value of natural gas at any point in time and at any network location. For the purpose of this paper, GSO is used to compute GBM problem solutions. The inputs include the static network model and a collection of time-dependent parameters. The static network model contains specifications for the nodes, pipes, and compressors on the system. In addition, we specify gNodes, which represent sellers or offtakers of gas on the system, more than one of which can correspond to a physical network node. These can represent any meter stations, and multiple gNodes can be used to represent different pricelquantity levels for the same customer.

The temporal parameters for the GSO solver include purchases, sales, bid and offer prices, flows corresponding to pre-existing contracts, minimum and maximum offtake curves of buyers, and minimum and maximum supply curves as described above. The temporal outputs are physical variables that include discharge pressure, power, compression ratios, suction and discharge pressure, and through flow for all compressors on the system; cleared purchases and sales for all customers participating in the market; nodal pressures; and pressures and flows at each end of all pipes in the system. In addition to the physical solution, a market solution is provided that includes the LTV at each node, as well as the dual variables corresponding to constraints on compressor power and discharge pressure.

To model rolling horizon based optimization, the GSO tool can accept initial conditions to initialize the problem and output the system state at any specified time, so that it can be used to optimize the subsequent rolling horizon optimization step. While the computational implementation of the GBM solution requires elliptic (i.e. time-periodic) boundary conditions, the real data that is used in our case study is aperiodic. In order to apply the computational tool, for each solve over a time horizon $[0, T]$, the problem is solved over an extended time horizon $[0, T+\tau]$ and the solution is then restricted back to $[0, T]$. The output state at time $T$ is subsequently used to initialize the solution on the next time horizon $[T, 2 T]$.

\section{Computational Case Study}

\subsection{The Physical Dataset}


The computational case study discussed in this section is based on data obtained from a planning model of a pipeline system in the United States and temporal SCADA data measured from the same system during February and March of 2014, a period when energy networks in the United States were particularly stressed due to Polar Vortex conditions. The process used to develop and validate the network model by comparing simulations against SCADA measurements is described in [9]. As explained in that paper, the network model was simplified from a model used for capacity planning, typically with steady-state optimization. The simplification relied on several assumptions. First, passive components and connections in the system such as valves were removed, and their status (open/closed) was used to determine any modifications to the topology. Second, although multiple compressor units make up a compressor station, the suction and discharge of the entire station occur through common headers. Thus, we modeled each compressor station as a single aggregate compressor with an aggregate power and assume that the individual compressor units can be controlled locally to maintain the desired discharge pressure of the entire station. The extracted subsystem is illustrated in Figure 2, and consists of 78 model nodes, 95 pipes with total length of 444.25 miles, and 4 compressor stations. Major inflow is at the suction of compressor 1 and main outflow is from node $\mathrm{X}$, with smaller offtakes throughout the system and at laterals. Inflow at compressor 1 and outflow at node $\mathrm{X}$ feed the upstream and downstream portions of the same pipeline, respectively. This network serves three power plants located at nodes labelled E, H and Q.

For each pipe, the physical parameters used were length, diameter, and friction factor, which were taken directly from the pipeline planning model. However, as explained in [9], the friction factor was scaled down by an engineering factor of 0.85 to compensate for pipe efficiency factors commonly used by commercial software packages but not considered in the reduced modeling approach.

The temporal network model consists of measurements from the SCADA system used for operation of the pipeline from which the test system model was extracted. This system provides hourly measurements of pressure (psig), temperature (degrees F), and volumetric flow (mcfh) out of the system at 31 metered custody transfer meter and check measurement locations, as well as average gas gravity and thermal content (mBTU/mmscfd). Check measurements at the 4 compressor stations include suction and discharge pressure (psig), suction and discharge temperature (degrees F), and volumetric through-flow (mcfh). Using this information, we computed mass flow (mmscfd) at each reduced network model location where flow leaves or enters the system and pressure at the slack node.

The temporal quantities of interest for our analysis in this paper are SCADA-based hourly incoming and outgoing flows that, combined with publicly available pricing information, we use to construct time dependent bid and offer price/quantity pairs.

\subsection{Economic Data and Assumptions}

As explained in Sec. 2.2 and 2.3, hourly buy/sell positions of buyer and sellers of natural gas within the modeled footprint are required as inputs into the GBM. At present, no statistical data of that kind exists. To compensate for this, we made several assumptions.

First, we assumed that the only controllable supply is located at the entry point of inflow into compressor 1 , which effectively represents all suppliers located upstream of the footprint. To test the physical and economic limitations of the modeled footprint, we assumed that controllable supply is unlimited in magnitude, up to engineering constraints at the inflow location.

Second, we considered four controllable demand locations - exit point $\mathrm{X}$ and three power plants at points E, H and Q. For power plants, we assumed that their demand for natural gas is capped at the level of actual hourly deliveries. As explained in the next section, depending on the experiment, the exit point was modeled both as capped at actual gas deliveries or as an unlimited gas buyer.

Depending on the experiment, we considered two sets of pricing assumptions. To assess physical deliverability of the system we assumed offer and bid prices for controllable supply and demand that were constant in time. Bid prices for all locations were set above the offer price and equal for all locations. Under this assumption, the GSO algorithm would maximize total deliveries to controllable demand and make no economic preference for individual delivery points.

For pricing and social welfare analyses described in the next section, we assumed that upstream supply was available at the corresponding regional pricing index obtained from the S\&P Global Market Intelligence service (S\&P Global).

For power plants, bids were based on ratios of Locational Marginal Prices (LMPs) at power plants' pricing nodes divided by their respective full load heat rates obtained from S\&P Global. These bids change hourly and effectively represent an estimate of power plants' willingness to pay for fuel given hourly prices they receive for selling electricity.

For the downstream exit point, we used two pricing points - the regional downstream price index applied 
to deliveries capped at the actual hourly level and an hourly price for incremental deliveries above actual levels. For incremental demand at the exit point under the price was set similarly to power plants' and based on hourly LMPs at an electric trading hub in the area served by the downstream portion of the pipeline. That LMP is divided by a factor of $8.5 \mathrm{Mcf} / \mathrm{MWh}$ which is a mid-point between a typical combined cycle power plant and a combustion turbine generating unit. The resulting bid approximates the hourly willingness to pay by a representative gas-fired generating unit served by a downstream pipeline segment.

Upstream and downstream natural gas pricing indices were based on relevant pipeline trading hubs with values obtained from S\&P Global. These prices change daily on weekdays only, prices for Friday apply also on Saturday and Sunday.

\subsection{Objectives and Design of Numerical Experiments}

The objectives of numerical experiments conducted with the use GSO applied to the above described dataset were to evaluate the potential effect of transient optimization on physical natural gas deliverability, on economic efficiency and to evaluate the relationship between LTVs and regional day-ahead prices. To meet these objectives, we conducted four experiments 1) Physical Base Case (PBC); 2) Throughput maximization (TM); 3) Economic Base Case (EBC) and 4) Social Welfare maximization (SWM). All 4 cases share the same network model parameters and hourly incoming and outgoing flow data. These cases differ in terms of which flows are controllable by GSO and the offer/bid values assigned to controllable flows. An unlimited Controllable Supply was placed at the upstream entry point in all four cases. Offer prices at the upstream entry point were set as shown in the table below:

\begin{tabular}{llll}
\hline PBC & TM & EBC & SWM \\
\hline \$2/Mcf & \$2/Mcf & $\begin{array}{l}\text { Upstream } \\
\text { zone index }\end{array}$ & $\begin{array}{l}\text { Upstream } \\
\text { zone index }\end{array}$ \\
\hline
\end{tabular}

Demand bids were placed at the downstream exit point and at three locations serving power plants. The maximum controllable demand for power plants was set in each hour matching their actual hourly gas use. Under the PBC and EBC cases, controllable demand for the exit point was set at actual hourly out-flows. Under the TM and SWM cases, controllable demand at the exit point was doubled in size.

Bid prices for controllable demand were defined as specified in the table below

\begin{tabular}{llll}
\hline PBC & TM & EBC & SWM \\
\hline Power Plants & & & \\
\hline \$3/Mcf & \$3/Mcf & LMP/HR & LMP/HR \\
\hline Downstream & Exit Point & & \\
\hline \$3/Mcf & \$3/Mcf & $\begin{array}{l}\text { Downstream } \\
\text { zone index }\end{array}$ & LMP/8.5 \\
\hline
\end{tabular}

In all four cases, GSO was set to maximize social welfare. In the $\mathrm{PBC}$ and $\mathrm{TM}$ cases, buyers were willing to pay $\$ 3 / \mathrm{Mcf}$ and a single seller was offering unlimited supply at $\$ 2 / \mathrm{Mcf}$. Selecting such parameters guarantees that the system would maximize the throughput effectively measured at a $\$ 1 / \mathrm{Mcf}$ spread between buyers' bid seller's offer. In the EBC and SWM cases, and social welfare maximization was made sensitive to market prices reflective of relative values placed on natural gas supply and electricity demand during the two-month period of February and March of 2014.

The purpose of the PBC Case was to set up a comparison point against which to measure the effect of the transient optimization under the TM case. At the same time, we use the output of the PBC case to assess the reasonableness of the optimization model against SCADA data. Indeed, unlike the benchmarking performed in [9] where simulations relied on actual compressor setting, in the $\mathrm{PBC}$ case compressor operations are established by GSO and are unlikely to match actual compressor settings used. However, it is important to compare optimization results to actual data to verify that optimized results are reasonable. The results of such comparisons are illustrated in Figure 3 for February 2014. The figure depicts hourly discharge compressor pressure values computed by GSO compared with SCADA measurements, and confirms that simulated values are within the range of observed values. Comparisons made for March of 2014, not presented here, also support that conclusion.

The purpose of the TM experiment was to find the maximum realizable throughput in the same pipeline segment over and above actually realized demand.

The objective of the EBC experiment was to compute Locational Trade Values (LTVs) associated with the Base Case flow regime by using the observed pricing data. Once the LTVs were obtained, it became possible to estimate an associated social welfare.

The objective of the SWM case was to maximize the throughput at the time of need. The need is reflected in the value of incremental demand based on the approximated willingness of downstream electric generators to pay for gas supplies. That was in contrast to the TM case in which the throughput was maximized assuming that it was always needed. In addition, under the SWM case we directly assessed the social welfare of the gas supply system under study, 
compared it to the social welfare estimated under the EBC case and thus obtained the economic effect of the GBM mechanism based on transient optimization techniques.

In our rolling horizon modeling, we optimized the system for a 48-hour horizon with a 24-hour periodicity. System conditions were equalized at the beginning of the first and at the end of the last optimization hour and the state of the system was unloaded at the end of the $24^{\text {th }}$ hour of the optimization horizon. That system state was used as an initial condition for modeling the subsequent 48-hour horizon. The 24-hour periodicity reflects the daily decision cycle. Selection of the 48-hour optimization horizon is a reasonable assumption reflecting the difficulty for power plants to estimate their willingness to pay for gas over a longer horizon.

\section{Analysis and Economic Implications}

A comparison of simulation results under the four cases described in the previous section are summarized in Table 1.

Table 1. Estimated Effects of GBM using Transient Pipeline Optimization

\begin{tabular}{rcc}
\hline \multicolumn{1}{c}{ February 2014 } & March 2014 \\
\hline \multicolumn{1}{c}{ Throughput increase } & & \\
\hline \multicolumn{1}{c}{$S W M-P B C$} & $12 \%$ & $14 \%$ \\
\hline $\begin{array}{l}\text { Price reduction at exit } \\
\text { point (EBC) }\end{array}$ & $28 \%$ & $9 \%$ \\
\hline $\begin{array}{l}\text { Increase in Social } \\
\text { Welfare (SWM - EBC) }\end{array}$ & $8 \%$ & $14 \%$ \\
\hline
\end{tabular}

As presented in that Table, using transient optimization methods have the potential to increase pipeline throughput by $12 \%-14 \%$ at the time of significantly constrained operations such as those under the Polar Vortex conditions during the winter of 2014. The daily and hourly throughput comparison between the PBC and TM cases for February 2014 are shown in Fig. 4 and Fig. 5, respectively. Results for March 2014, although not shown, look similar and confirm the above conclusion.

Hourly and daily delivery dynamics shown in these two figures indicate that the maximum achievable throughput changes over time. Although maximized deliveries are reflective of system capacity, the latter appears to be dependent on system conditions.

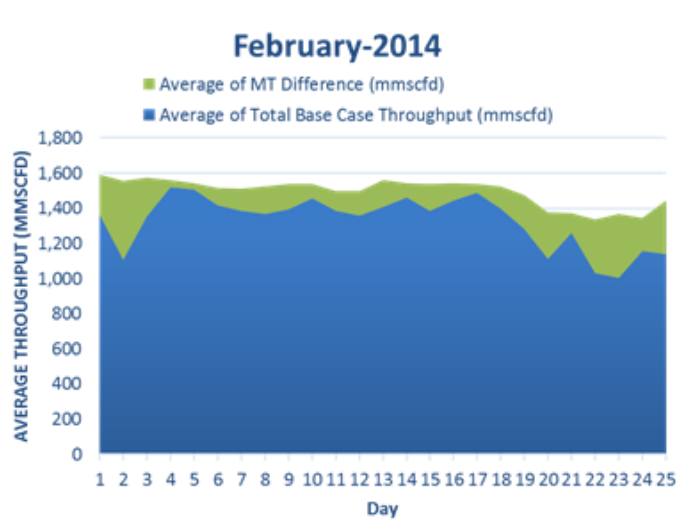

Figure 4. Daily Effect to Throughput Optimization (Feb-2014)

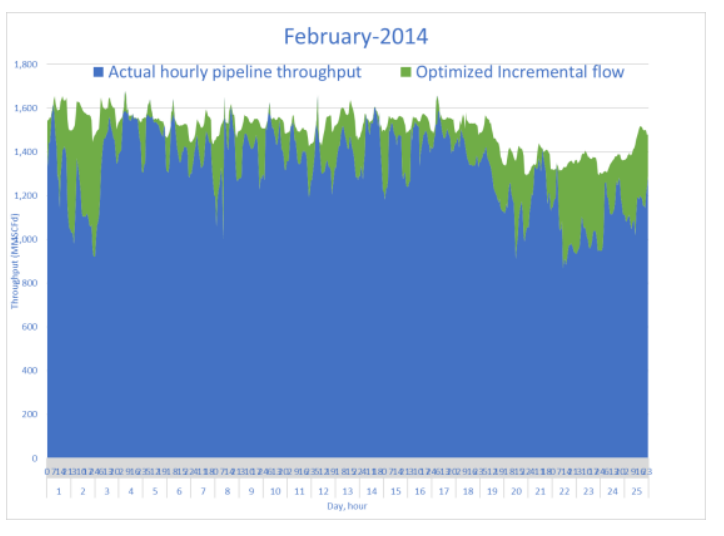

Figure 5. Hourly Effect of Throughput
Optimization (Feb-2014)

As one would expect, optimized incremental deliveries appear to be bigger in magnitude at the time of lower deliveries under the PCB case. This observation indicates that incremental throughput may appear at times when it is not needed and the $12 \%-14 \%$ increase in throughput may be overstating the economic effect of transient optimization.

The EBC and SWM cases designed to address these concerns indicate that the magnitude of the incremental throughput at the time of need is in the range of between $7 \%$ and $9 \%$. The need here is measured in terms of the estimated willingness of power generators located downstream to pay for gas relative to the gas supplier offer price.

The 7\% - 9\% effect is smaller than under the TM PBC comparison, but is still very significant considering that it is measured under extremely constrained historical operational conditions.

We estimate a $7 \%$ - $8 \%$ increase in social welfare of the operation of the pipeline segment under study attributable to the modeled implementation of GBM. By analyzing LTV values under the EBC scenario 
shown daily in Fig. 6 and hourly in Fig. 7. This comparison indicates that using GBM-based LTVs as a pricing mechanism for natural gas could have significantly reduced natural gas prices to consumers and at the same time electricity production costs and resulting electricity prices. The noted difference between the zonal price index and LTVs indicates that most of the time, LTV serves as price cap - that is particularly visible in Figure 7. On any day, under the EBC scenario flows are constrained only in a few hours at most. As a result, only in these hours LTVs hit the price cap. The magnitude of price reduction in March appears much smaller than in February. This is simply because that March prices were less volatile than in February resulting in lower average price differences.

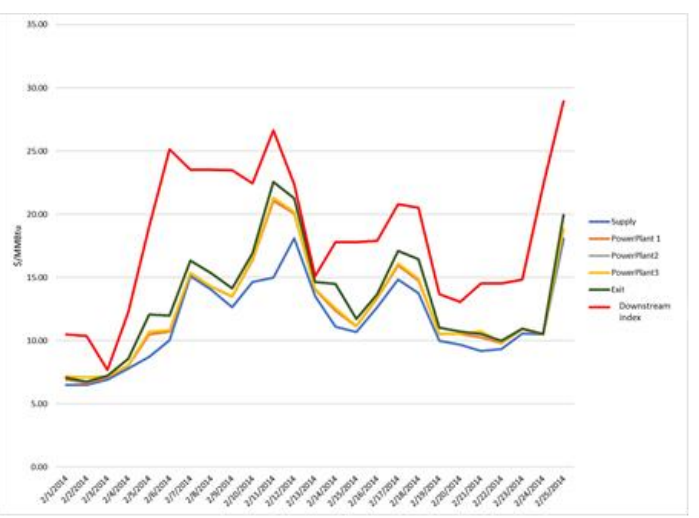

Figure 6. LTV vs. Downstream Price Index - Daily Comparison

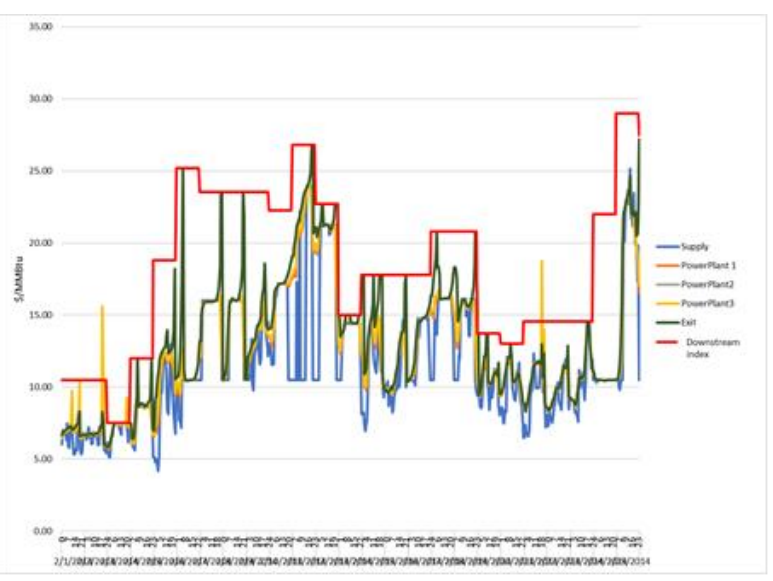

Figure 7. LTV vs. Downstream Price Index, Hourly Comparison

The above analyses are performed under intentionally narrow but realistic assumptions, constrained by the economic data available. As a result, we considered a very narrow application of the
GBM limited to a very few locations on the pipeline system - an entry point, an exit point and three power plant locations. No flexibility and trading opportunities were assumed for supply and deliveries for all other points due to the lack of underlying economic data. Our conjecture is that the effect of the GBM based on transient pipeline optimization may be significantly bigger if the scope of market participants was more broadly spanned over the pipeline network resulting in greater elasticity of supply and demand in the system.

\section{Conclusions}

The focus of this paper was to review the Gas Balancing Market (GBM) design and present a case study based on historical data of a pipeline subsystem in the United States. The proposed GBM is structured to complement the timing and logic of the existing US natural gas markets. It is specifically designed to operate in the time period when there is no other formal market mechanism. The goal of the GBM is to improve the economic and operating efficiencies of both the natural gas and the electric markets. The fact that the GBM follows the standard gas day market and is a voluntary, platform-based bilateral market means that the GBM requires no changes in today's operational markets and may not require more than standard market oversight, i.e., is not likely to require FERC approval for its establishment.

The economic implications of the establishment of a GBM are significant as discussed in Section 4. In the case study presented for a real pipeline subsystem we show that at a time of constrained delivery capability because of extreme weather conditions, the GBM structure would have allowed for an increase in natural gas throughput of $12 \%$ to $14 \%$. The impact on the electric market during this time period would have been a decrease in cost natural gas costs as a fuel for electric generation of $14 \%$ to $28 \%$, with an effect on electricity prices of the same order of magnitude.

The GBM is proposed to operate on a pipeline by pipeline basis or potentially begin with operation on a constrained segment or end of a pipeline. As a result, the GBM would explicitly not require any type of regional organization to coordinate its operations. A GBM could be instituted at a single pipeline level and could also be set to serve only a part of the pipeline system, to simplify an initial implementation.

Participation in the GBM would be entirely voluntary. The bilateral, auction-based structure provides the opportunity of participation and additional revenue through the clearing mechanism but is not obligatory relative either to the physical operation of the pipeline or of the economic benefits that can 
accrue. Participants will enter and leave, buy and sell if and when the benefits of participation exceed the costs of participation.

Participation in the GBM offers benefits to market participats both on the gas side and on the electric side. On the gas side, the GBM will provide relief of pipeline constraints through LTV-sensitive optimization of compressors helping to determine the most efficient line pack strategy over time and across the system to assure higher delivery to locations with the highest value of gas. At the same time, redispatch of electric generation in response to dynamically formed LTVs will provide additional relief of pipeline constraints.

In the long run we have shown that the creation of the GBM will help the pipeline and their customers make better operating and investment decisions.

\section{References}

[1] "Federal Energy Regulatory Commission (FERC) order \#787," www.ferc.gov/CalendarFiles/20131115164637RM13-17-000.pdf.

[2] "Federal Energy Regulatory Commission (FERC) order \#809,"http://www.ferc.gov/whats-

new/commmeet/2015/041615/M-1.pdf

[3] MITEI. (2013) Growing concerns, possible solutions:

The interdependency of natural gas and electricity systems. [Online]. Available: http://mitei.mit.edu/publications/ reports-studies/growing-concerns-possible-solutions [4] E. Litvinov, "Design and operation of the locational marginal prices-based electricity markets," Generation, Transmission Distribution, IET, vol. 4, no. 2, pp. 315-323, February 2010.

[5] Forward Market Operations, "Energy \& Ancillary Services Market Operations, M-11 Rev. 75," PJM, Tech. Rep., 2015.
[6] A. Rudkevich, and A. Zlotnik. "Locational Marginal Pricing of Natural Gas subject to Engineering Constraints." Proceedings of the 50th Hawaii International Conference on System Sciences, pp. 3092-3101, 2017 [7] E. G. Read, B. J. Ring, S. R. Starkey, and W. Pepper, "An LP-based market design for natural gas," in Handbook of networks in power systems II. Springer, 2012, pp. 77-113.

[8] A. Zlotnik, A. Rudkevich, M. Vuffray, S. Misra, "Dynamic Marginal pricing for Optimal Scheduling of Natural Gas Supply and Delivery over a Pipeline Network," In preparation

[9] A. Zlotnik, A. Rudkevich, E. Goldis, P.A. Ruiz, M. Caramanis, R. Carter, S. Backhaus, R. Tabors, R. Hornby, D. Baldwin. "Economic Optimization of Intra-Day Pipeline Flow Schedules using Transient Flow Models." Proceedings of the PSIG 2017 Conference, Atlanta, GA, May.

[10]. E. S. Menon, Gas pipeline hydraulics. CRC Press,

2005.

[11]. A. L. Lee, M. H. Gonzalez, and B. E. Eakin. "The viscosity of natural gases." Journal of Petroleum Technology vol. 18 , no. 8 , pp. 997-1000, 1966

[12]. A. Zlotnik, M. Chertkov, and S. Backhaus, "Optimal control of transient flow in natural gas networks," in 54th IEEE Conference on Decision and Control, Osaka, Japan, 2015, pp. 4563-4570.

[13]. A. Osiadacz. "Simulation of transient gas flows in networks." International journal for numerical methods in fluids 4.1 (1984): 13-24.

[14]. A. Rudkevich, A. Zlotnik, P. Ruiz, E. Goldis, R. Tabors, R. Hornby, S. Backhaus, M. Caramanis, A. Beylin, R. Philbrick. Market Based Intraday Coordination of Electric and Natural Gas System Operation. In.: Proceedings of the $51^{\text {st }}$ Hawaii International Conference on System Science, 2018. 


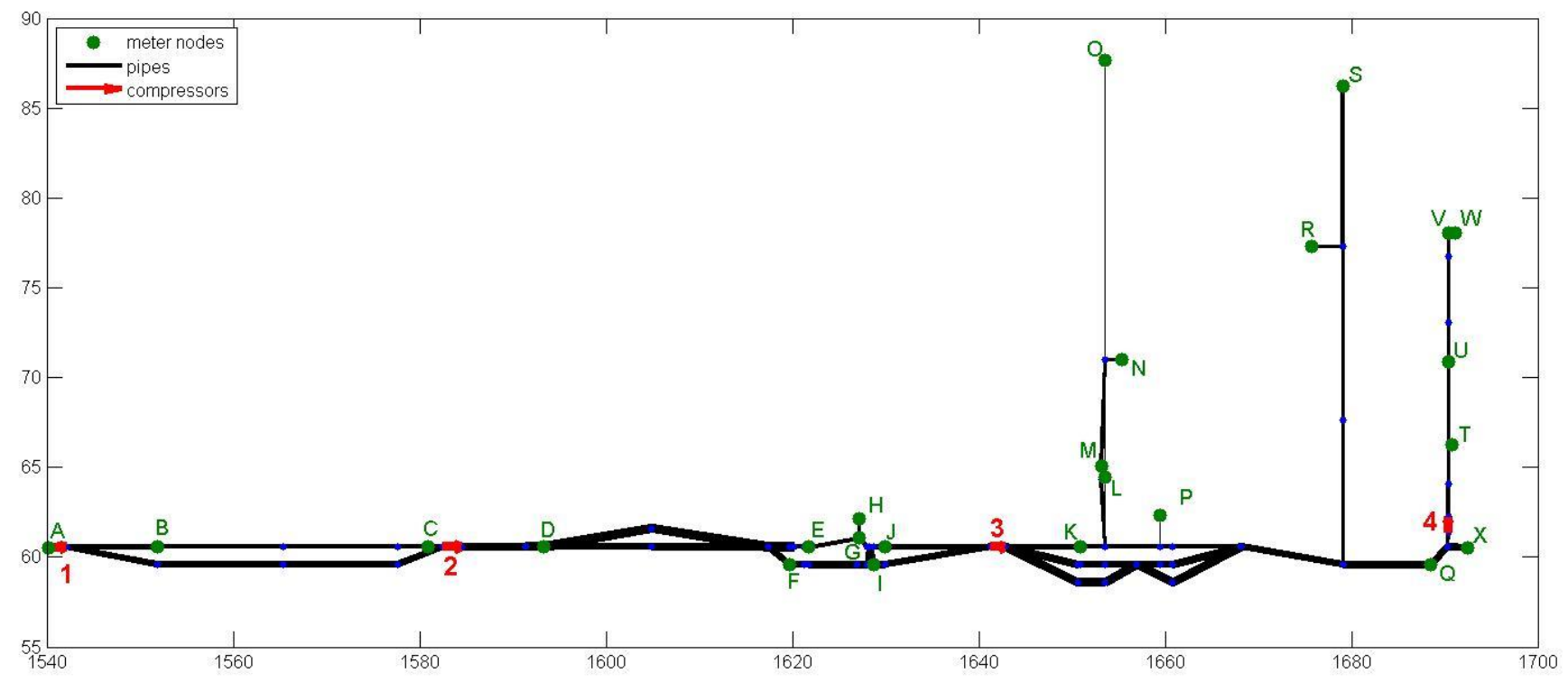

Figure 2. Pipeline Network Diagram

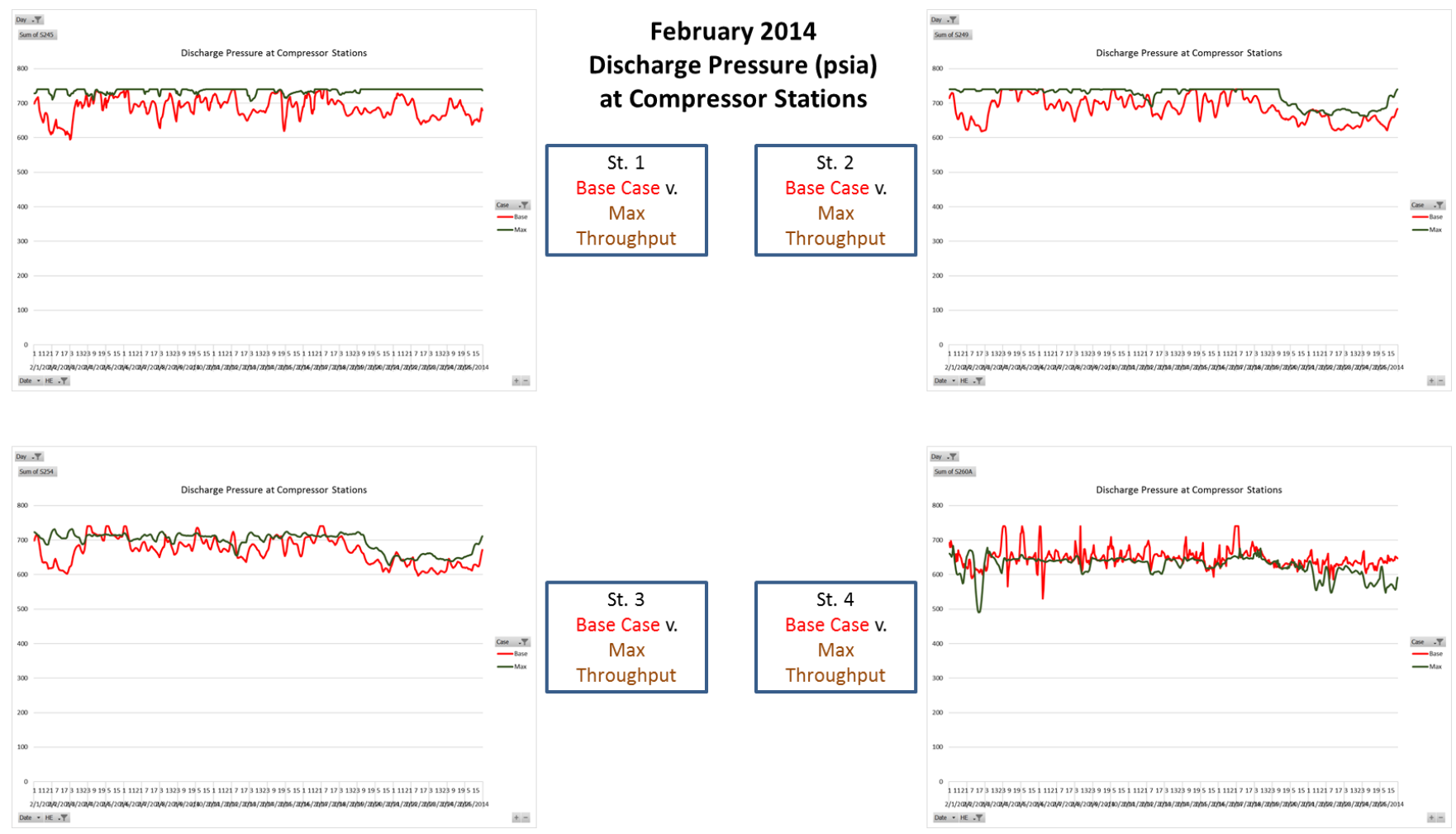

Figure 3. Compressor Settings Simulated vs. Actual, February 2014 